\title{
Laparoscopic Inguinal Hernia Repair with Closure of Hernial Defect and Central Mesh Fixation Using Glubran 2
}

\author{
Ahmed E. Lasheen*, Adel M. Tolba, Hany Mohamed, Hatem Mohammed, Nadia A. Smaeil \\ General Surgery Department, Faculty of Medicine, Zagazig University, \\ Egypt and National Hospital, Riyadh, Saudi Arabia \\ Email: *lasheenahmed@yahoo.com
}

Received November 2, 2013; revised December 2, 2013; accepted December 10, 2013

Copyright (c) 2013 Ahmed E. Lasheen et al. This is an open access article distributed under the Creative Commons Attribution License, which permits unrestricted use, distribution, and reproduction in any medium, provided the original work is properly cited.

\begin{abstract}
Background: Laparoscopic tension-free repair of inguinal hernia was presented in 1990s, promising less pain and short recovery period, but carrying the risk mesh bulging and migration. Objective: We have presented our technique in which central zone of mesh is fixed only after closure of hernial defect. Patients and Methods: This study included 27 males patients (14 indirect inguinal hernias, 9 direct inguinal hernias, 4 both direct and indirect inguinal hernias on the same side). These cases are undergoing tension-free mesh repair after closure of hernial defect, and the mesh is fixed only at its central zone using Gulbran 2, between April 2011 and March 2013. The follow-up period ranged from 6 to 30 months. The intra and postoperative complications were recorded. Results: Mean hospital stay was 1 day. The age of this group of patients ranged from 23 to 63 years (mean, 47 years). The operative time ranged from 30 to 100 minutes (mean, 45 minutes). The intraoperative complications were in form of mild bleeding in 7 patients (25.9\%) during hernial sac dissection. Postoperative complications were mild inguinal pain in 4 patients (14.8\%) for three weeks. Mild hydrocele in 3 patients (11\%) was recorded. No recurrence or bulging at hernia site was noticed during the period of follow-up. Conclusion: Laparoscopic inguinal hernia repair with central mesh fixation after closure of hernial defect is effective, easy and free of complications.
\end{abstract}

Keywords: Laparoscopic Hernia Repair; Defect Closure; Mesh Fixation Glubrane 2

\section{Introduction}

Herniorraphy is the second prevalent operation after appendectomy in general surgery [1]. The standard method for inguinal hernia repair had been changed little over a hundred years until the introduction of synthetic mesh. This mesh can be placed as either an open or a minimally invasive endoscopic technique. The most common endoscopic techniques are transabdominal preperitoneal (TAPP) [2] and totally extraperitoneal (TEP) [3] approaches. Classical mesh fixation using tacher may be etiology of postoperative pain, bladder injury and major blood vessels injury [4]. Some surgeons advocated placing the mesh without fixation (tension-free mesh repair) in the preperitoneal space to avoid these complications, but carrying the risk of mesh bulging or migration [5,6]. In this study, the central zone (about central one third) of

${ }^{*}$ Corresponding author. mesh corresponding iliopubic tract is fixed only by using Glubran 2, after closure of hernial defect.

\section{Patients and Methods}

This study was included 27 males patients (14 indirect inguinal hernias, 9 direct inguinal hernias, 4 both direct and indirect inguinal hernias on same side). All information about the technique was discussed with all patients, and all patients gave writing consent for inclusion of their data in this study. The age of the patients ranged from 23 to 63 years (mean, 47 years).

Surgical technique: Under general anesthesia, the laparoscopic transabdominal preperitoneal (TAPP) technique is used in this patients group. The patient asked to urinate, while in the surgical preparation room, in order to empty the bladder. The Veress needle is inserted supra-umbilically to install the pneumoperitoneum of 14 mmHg has been achieved. A 0-degree, $10 \mathrm{~mm}$ laparo- 
scope is used for the whole procedure. Two additional trocars of $5 \mathrm{~mm}$ in diameters, one on the right side of the patient, at the level of umbilicus, lateral to the rectus sheath, and another one, on left side of the patient, slightly below the umbilicus, lateral to the rectus sheath. The patient is in supine, Trendelenburg position, with the right arm along the body and venous access on the left arm. The surgeon takes up a position on the right side of the patient and assistant and scrub nurse on the left. As usually, the peritoneum is incised superiorly, three to four $\mathrm{cm}$ above the hernia defect. This incision extends from the medial umbilical ligament to the anterior superior iliac spine laterally. After dissection of hernia sac and complete exposure of the preperitoneal space, the hernia defect was closed helping spinal needle (No. 22G) percutaneously and using prolene No. $2 / 0$, and the suture is tied extracorporeal or intracorporeal (Figures 1(a)-(e)). Suitable mesh is fixed at central zone (about one third) only corresponding from anterior superior iliac spine to the symphysis pubis using Glubran 2 (Gem srl, Viareggio, Italy) Figures 2(a)-(c). The peritoneum is closed with using Vicryl No. 2/0, and the mesh remains in a completely extraperitoneal position. The pneumoperitoneum is emptied under direct viewing with the laparoscope and external pressure is applied to the inguinal region. The trocar sites are closed and an elastic support for scrotal compression is placed. The follow up period ranged from 6 to 30 months (mean, 22 months). The intra and postop-

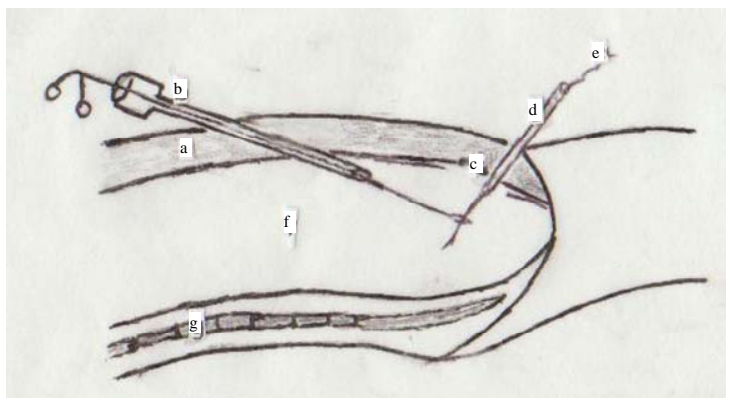

(a)

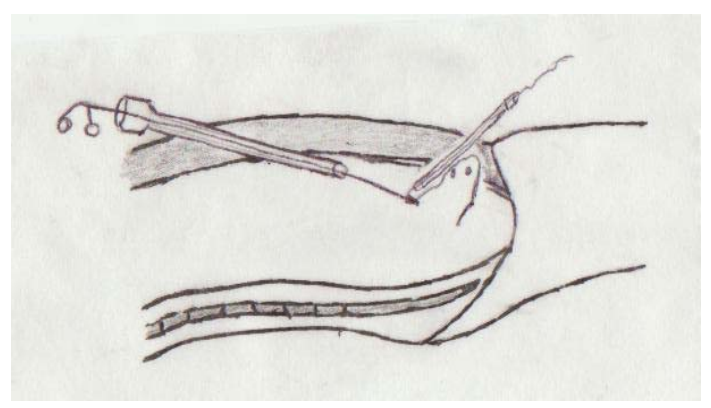

(c)

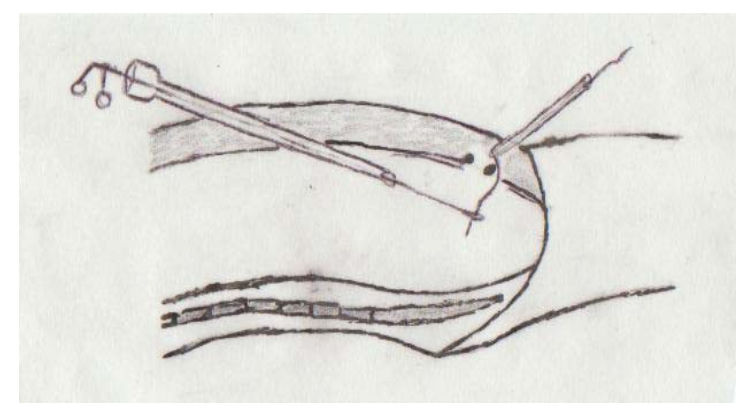

(b)

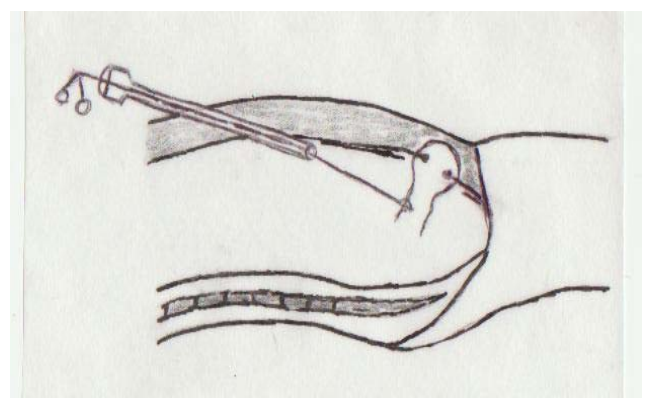

(d)

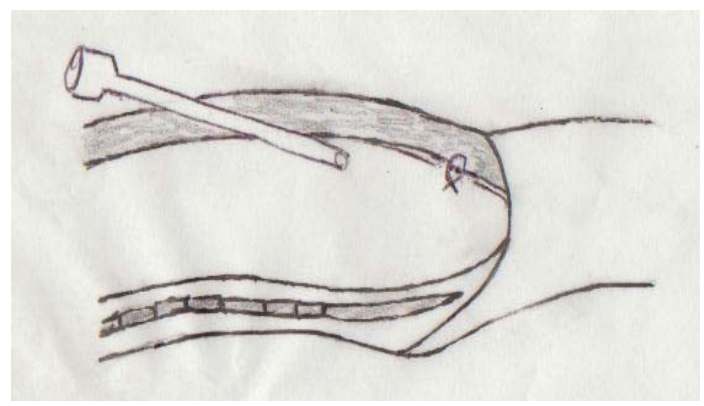

(e)

Figure 1. (a) a-Anterior abdominal wall. b-Trocar port $5 \mathrm{~mm}$. c-Hernia defect. d-Spinal needle No. 20G. e-Prolene No. 2/0 inside spinal needle. f-Peritoneal cavity. g-Posterior abdominal wall. The spinal needle and prolene No. 2/0 inside it passed under the lower edge of hernia defect to peritoneal cavity. The prolene end was holed from peritoneal cavity by grasper. (b) The spinal needle was partial withdraw and redirected to pass to peritoneal cavity above the upper edge of hernia defect with prolene thread. (c) The spinal needle and prolene inside it passed to peritoneal cavity above the upper hernia edge. Then, the other prolene end was holed by grasper. (d) The spinal needle was removed through prolene holding by grasper. One suture is formed to pass under the lower edge of hernia defect, floor of defect, and above the upper edge of hernia defect. (e) The suture was tied extracorporeal or intracorporeal to close the hernia defect. 


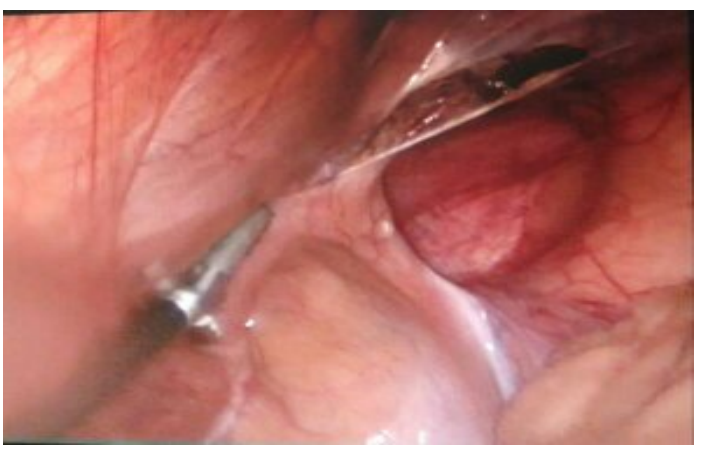

(a)

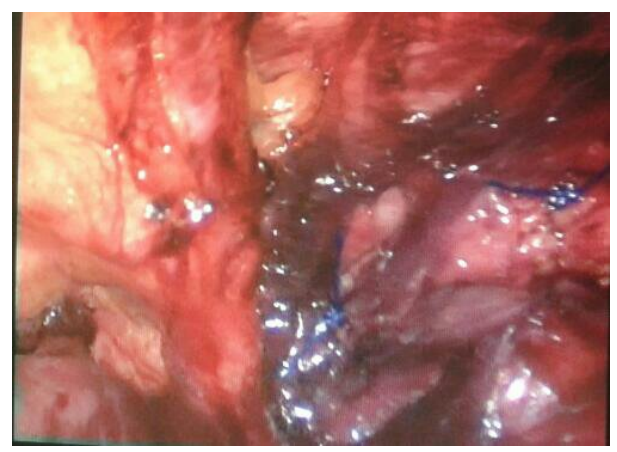

(b)

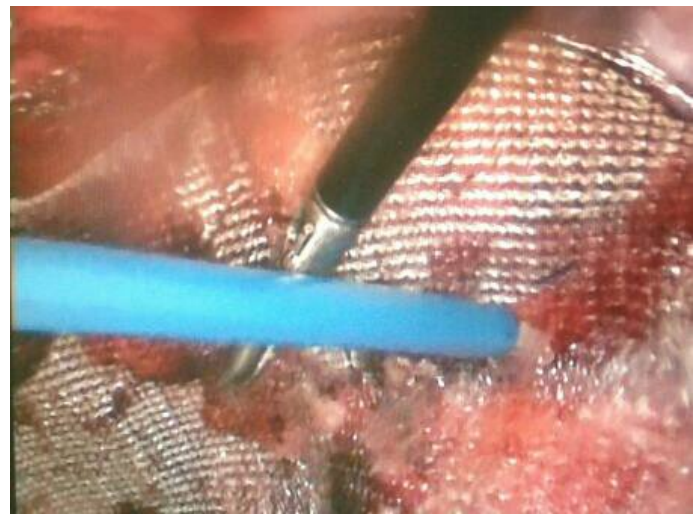

(c)

Figure 2. (a) One patient suffered from both (direct and indirect) types of inguinal hernia on right side and the dissection of sacs started. (b) The hernia defects were closed by two sutures using prolene No. 2/0. (c) The central zone (about central one third) of mesh corresponding to iliopubic tract was fixed to underlining tissue using Glubran 2.

erative complications were recorded.

\section{Results}

The mean surgical time was 45 minutes (ranged from 30 to 100 minutes). Twenty two patients (81.5\%) of the cases returned to their usual activities in one week and five patients (18.5\%) required up two weeks. Hydroceles developed in three cases (11\%), after correction of indirect inguinal hernias, which were solved with a single aspiration. Four patients (14.8\%) experienced mild inguinal pain for three weeks. Mild bleeding occurred during dissection of hernia sac from cord in 7 patients (25.9\%) which, controlled with cauterization. No recurrence or bulging of the mesh were recorded during the period of follow up.

\section{Discussion}

The increased acceptance of inguinal hernia repair by a laparoscopic approach has led to many reports confused reports on technique, results, and complications related to this procedure. Many of these complications are directly related to lack of thorough knowledge of surgical anatomy or improper technique $[7,8]$. The complication rates after laparoscopic hernioplasty vary from $5 \%$ to $13 \%$, but the definition of complications differs widely among studies $[9,10]$. Severe bleeding usually due to vascular injury of iliac vessels and generally occurs because the using of staples during mesh fixation [11]. Patients who undergo laparoscopic herniorraphy have $1.6 \%$ incidence of neuralgias due to nerve entrapment during mesh fixation with staples [12]. Some surgeons advocated placing the mesh without fixation in preperitoneal space to avoid nerve injury [13]. In our technique, the central zone of the mesh only fixed by using Glubrane 2 substance corresponding to iliopubic tract, preventing any injury to nerves and vessels. Mesh bulging was considered a failure of the correct surgical technique to tightly stretch the mesh over the hernia opening. Two factors are predisposed for mesh protrusion a loosely stretched mesh and present of hernia defect [5]. The mesh in our technique is fixed at central zone after closure of hernia defect to allow the mesh expanded peripherally when the pneumoperitoneum is released. The recorded recurrence rate is $0.1 \%$ following TAPP. The main cause of recurrence is mesh not adequately fixed and presence of hernia defect allowing the mesh to migrate through it [11]. The recurrence rate in our study is zero, this due to closure of hernia defect behind the mesh, which fixed adequately over the closed hernial defect. 


\section{Conclusion}

Our technique for laparoscopic inguinal hernia repair is easy, associated with good results and free of complications.

\section{REFERENCES}

[1] L. J. Grunwaldt, S. D. Schwaitzberg, D. W. Rattner and D. B. Jones, "Is Laparoscopic Inguinal Hernia Repair an Operation of the Past?" Journal of the American College of Surgeons, Vol. 200, No. 4, 2005, pp. 616-620. http://dx.doi.org/10.1016/j.jamcollsurg.2004.10.033

[2] R. Bittner, C. G. Schmedt, J. Schwarz, K. Kraft and B. J. Leibl, "Laparoscopic Transperitoneal Procedure for Routine Repair of Groin Hernia,” British Journal of Surgery, Vol. 89, No. 8, 2002, pp. 1062-1066. http://dx.doi.org/10.1046/j.1365-2168.2002.02178.x

[3] S. Bringman, S. Ramel, T. J. Heikkinen, T. Englud, B. Westman and B. Anderberg, "Tension-Free Inguinal Hernia Repair: TEP versus Mesh-Plug versus Lichtenstien: A Prospective Randomized Controlled Trial," Annals of Surgery, Vol. 237, No. 1, 2003, pp. 142-147. http://dx.doi.org/10.1097/00000658-200301000-00020

[4] E. A. O’Reilly, J. P. Burke and P. R. O’Connell, “A MetaAnalysis of Surgical Morbidity and Recurrence after Laparoscopic and Open Repair of Primary Unilateral Inguinal Hernia,” Annals of Surgery, Vol. 255, No. 5, 2012, pp. 846-853.

http://dx.doi.org/10.1097/SLA.0b013e31824e96cf

[5] E. J. Schoenmaeckers, E. B. Wassenaar, J. T. Raymakers and S. Rakic, "Bulging of the Mesh after Laparoscopic Repair of Ventral and Incisional Hernias,” Journal of the Society of Laparoendscopic Surgeons, Vol. 14, No. 4, 2012, pp. 541-546.

http://dx.doi.org/10.4293/108680810X12924466008240
[6] S. Yakan, A. Coskun, A. Senlikci, M. A. Ustuner, M. Yildirim and N. Erkan, "Mesh Migration into the Lumen of the Small Bowel. A Late Complication of Incisional Hernia Repair,” Surgical Chronicles, Vol. 2, 2013, pp. 102-103.

[7] M. S. Kavic, “Laparoscopic Hernia Repair,” Surgical Endoscopy, Vol. 9, No. 1, 1995, pp. 12-15. http://dx.doi.org/10.1007/BF00187877

[8] N. Kosai, P. A. Sutton, J. Evans and J. Varghese, "Laparoscopic Preperitoneal Mesh Repair Using a Novel SelfAdhesive Mesh,” Journal of Minimal Access Surgery, Vol. 7, No. 3, 2011, pp. 192-194.

[9] R. Fitzgibbons, J. Camps, D. Comet, N. Nguyen, B. Litke, R. Annibali and G. Salerno, "Laparoscopic Inguinal Herniorrhaphy Results of a Multicenter Trial,” Annals of Surgery, Vol. 221, No. 1, 1995, pp. 3-113. http://dx.doi.org/10.1097/00000658-199501000-00002

[10] J. Payne, "Complications of Laparoscopic Inguinal Herniorrhaphy,” Seminars in Laparoscopic Surgery, Vol. 4, 1997, pp. 166-181.

[11] P. Miguel, M. Reusch, A. daRosa and J. Carlos, "Laparoscopic Hernia Repair-Complications,” Journal of the Society of Laparoendscopic Surgeons, Vol. 2, No. 1, 1998, pp. 35-40.

[12] R. Annibali and R. J. Fitzgibbons, "Surgical Anatomy of the Inguinal Region and Lower Abdominal Wall: The Laparoscopic Perspective," 80th Annual Clinical Congress, American College of Surgeons, Chicago, 1994, pp. 72-85.

[13] A. Hakeem and V. Shanmugam, "Effectiveness of Multiple Neurectomies to Prevent Chronic Groin Pain after Tension-Free Hernia Repair,” International Surgery, Vol. 96, No. 2, 2011, pp. 162-163. http://dx.doi.org/10.9738/1359.1 